\title{
Part II: Fibroblasts preferentially migrate in the direction of principal strain
}

\author{
G. P. Raeber · M. P. Lutolf · J. A. Hubbell
}

Received: 9 December 2006 / Accepted: 25 May 2007 / Published online: 7 July 2007

(C) Springer-Verlag 2007

\begin{abstract}
A growing body of evidence suggests that the sensory information from the cytoskeleton and integrins may be responsible for guiding migration during mechano- and haptotaxis. However, the dual function of these subcellular structures as mechano-sensors and -actuators is only partially understood. Using a new cell chamber described in the preceding companion paper (Ref to part I, Raeber et al. 2007a) we investigated the migration response of adhesiondependent fibroblasts embedded 3-dimensionally within synthetic protease-sensitive poly(ethylene glycol) hydrogels to stepwise and cyclic mechanical loads. To that end, we developed a spatially and temporally resolved migration analysis technique capable of providing estimates of statistical cell migration parameters along and perpendicular to the main strain direction. Fibroblasts reoriented themselves in the direction of principal strain, increased their proteolytic migration activity and moved preferentially parallel to the principal strain axis. These results point to a possible correlation between planes of iso-strain and migration direction.
\end{abstract}

Keywords Mechanical stimulation - Cell migration . Local effective stiffness · PEG hydrogels

G. P. Raeber · M. P. Lutolf · J. A. Hubbell ( $ه)$

Institute of Bioengineering, EPFL, LMRP, Station 15,

1015 Lausanne, Switzerland

e-mail: jeffrey.hubbell@epfl.ch

G. P. Raeber

Institut Straumann AG, Basel, Switzerland

J. A. Hubbell

Institute of Chemical Sciences and Engineering,

Ecole Polytechnique Fédérale de Lausanne (EPFL),

Lausanne, Switzerland

\section{Introduction}

Mechanical signaling plays a pivotal role in both physiological and pathological development of many tissues, including the central nervous system (Van Essen 1997), connective tissue(Tomasek et al. 2002), bone (Duncan and Turner 1995), and vascular tissue (Williams 1998). Likewise, mechanical cues have been shown to regulate morphogenesis (Henderson and Carter 2002), angiogenesis (Ingber 2002), migration (Goldmann 2002; Li et al. 2003; von Offenberg Sweeney et al. 2004), gene expression (Shyy and Chien 1997), and stem cell differentiation (Engler et al. 2006). Therefore, besides chemical signals, biophysical signals have recently received increased consideration in various fields of biomedicine.

By the use of model substrates with controlled chemical composition, several studies have succeeded in showing that cells respond to the mechanical stiffness of the extracellular matrix (ECM) (Balaban et al. 2001; Choquet et al. 1997; Katz et al. 2000; Lo et al. 2000; Engler et al. 2006) and the area of the substrate that is available for binding (Choquet et al. 1997). The conversion of such biophysical stimuli into cellular responses is generally referred to as mechanotransduction. Several distinct cellular structures have been proposed as mechanosensors, including integrins (Katsumi et al. 2004), ion channels (Sachs and Morris 1998), and the cytoskeleton (Ingber 1997). However, the understanding of the precise function of these mechanosensing complexes and how they choreograph related signaling pathways remains an outstanding challenge in cell biology.

The cytoskeleton and integrins are not only involved in withstanding and sensing mechanical loads, they also generate and transmit physical forces. Concerted action of actinomyosin-driven contraction and integrin-mediated adhesion enables cell migration and is responsible for the intrinsic tensional homeostasis in many tissues. Integrins 
cluster when forces develop at their site and eventually form focal adhesions in the case of sustained tension (Geiger and Bershadsky 2001). Concomitantly, structural reinforcement of the adhesion site, strengthening of ECM-cytoskeleton linkage and strong signaling activity sets in (Balaban et al. 2001; Choquet et al. 1997; Koo et al. 2002; Riveline et al. 2001; Wang et al. 1993). Focal adhesions are characteristic for cells adhering to a surface. However, in a three-dimensional (3D) environment cells develop similar cell-matrix contacts that presumably possess mechanosensory functions as well (Cukierman et al. 2001, 2002).

Importantly, the forces that induce intracellular signaling via mechanoreceptors may originate from external loads (balanced by the cytoskeleton of the cell) or from the contractile apparatus of the cell itself (balanced by the ECM or neighboring cells). In both cases, the mechanical properties of the ECM and the cell are pivotal, since they determine the time-dependent stresses that develop at mechanoreceptive sites. In view of these considerations, the quantitative measurement of $3 \mathrm{D}$ cell migration in a controlled static or dynamic mechanical environment is of considerable interest.

The matrix that transmits loads to the cells and mimics the natural ECM is of crucial importance for in vitro mechanobiological investigations targeting such questions. Synthetic poly (ethylene glycol) (PEG) hydrogels are a novel class of biomaterials that have been shown to be useful as model matrices for the quantitative investigation of proteolytic cell migration (Raeber et al. 2005) and as instructive microenvironments for morphogenesis in tissue engineering (Lutolf and Hubbell 2005). PEG macromers serve as an inert structural network, held together by proteasesensitive peptide sequences and decorated by pendant adhesion ligands. Hence, the biologically active peptides render the otherwise 'stealth' hydrogels cell-adhesive and degradable by cell-sequestered enzymes. Their purely synthetic nature offers the possibility to systematically reconstitute a specific extracellular environment on a molecular level, thereby allowing for tailor-made physical and chemical properties. Of particular interest in mechanobiology is the almost linear elastic material behavior (e.g. negligible creep) since it ensures constant physical properties during long-term static or dynamic mechanical stimulation.

Building on the previously developed uniaxial cell stimulation device (UCSD), described in the preceding companion paper (Ref to part I, Raeber et al. 2007a) our target in this work was to quantitatively investigate the proteolytic migration response of human fibroblasts embedded in matrix metalloproteinase (MMP)-sensitive PEG hydrogels to mechanical stimulation. Fibroblasts were used as a prototypical cell, known to be involved in the mechano-regulated remodeling of soft connective tissue (Tomasek et al. 2002). We extended our previous migration analysis technique to enable evaluation of migration along and perpendicular to the principal strain axis and subjected the cells to two prototypical stimulation regimes. Our results provide evidence that mechanical stimulation induces proteolytic cell migration in the direction of principal strain, which is sustained after unloading.

\section{Materials and methods}

\subsection{PEG derivatives and peptides}

Branched 4arm-PEG macromers, $20 \mathrm{kDa}$, were purchased from Shearwater Polymers (Huntsville, AL, USA) and functionalized at the OH-terminus. Divinyl sulfone was from Aldrich (Buchs, Switzerland). Synthesis and purification of PEG vinyl sulfones (PEG-VS) and peptides is described in the preceding companion paper (Ref to part I, Raeber et al. 2007a). All standard peptide synthesis chemicals were analytical grade or better and were purchased from Novabiochem (Läufelfingen, Switzerland).

\subsection{Cell culture}

Human foreskin fibroblasts (HFFs; neo-natal normal human dermal fibroblast; Clonetics, San Diego, CA, USA) were cultured in fibroblast cell culture medium (Dulbecco's Modified Eagle's Medium, with 10\% fetal bovine serum (FBS) and 1\% antibiotic-antimycotic, all Gibco BRL, Life Technologies, Grand Island, NY, USA) at $37^{\circ} \mathrm{C}$ and $5 \% \mathrm{CO}_{2}$. Cells were removed from culture substrates using $0.05 \%$ trypsin $/ 0.02 \%$ EDTA (Gibco BRL), centrifuged at $400 \mathrm{~g}$ for $5 \mathrm{~min}$, resuspended in culture medium, and added to the hydrogel precursor. For all experiments, cells from subconfluent cultures at passages P9 and P10 were used in conjunction with the same FBS batch. Medium was replaced every three days. To that end, the closed loop of the medium circulation was opened and the chamber volume was exchanged with $50 \mathrm{ml}$ pre-warmed fresh medium.

\subsection{Casting of PEG hydrogel constructs}

PEG hydrogel precursor solutions contained $\sim 32 \mu \mathrm{M}$ integrin-binding peptides Ac-GCGYG RGDSPG (adhesion domain shown in italic) (Hern and Hubbell 1998; Pierschbacher and Ruoslahti 1984) and were rendered MMPdegradable by crosslinking the PEG macromers with a bifunctional MMP-sensitive peptide sequence (e.g. Ac-GCRD-GPQG $\downarrow$ IWGQ-DRCG, where $\downarrow$ indicates the cleavage site) (Lutolf et al. 2003a). The PEG materials used herein had a precursor concentration of $10 \%$ and a stoichiometric ratio of 0.83 (molar ratio of VS and SH groups, see Lutolf and Hubbell 2003). HFFs were added to the PEG precursor solution and had a concentration of $10^{5}$ cells $/ \mathrm{ml}$ after 


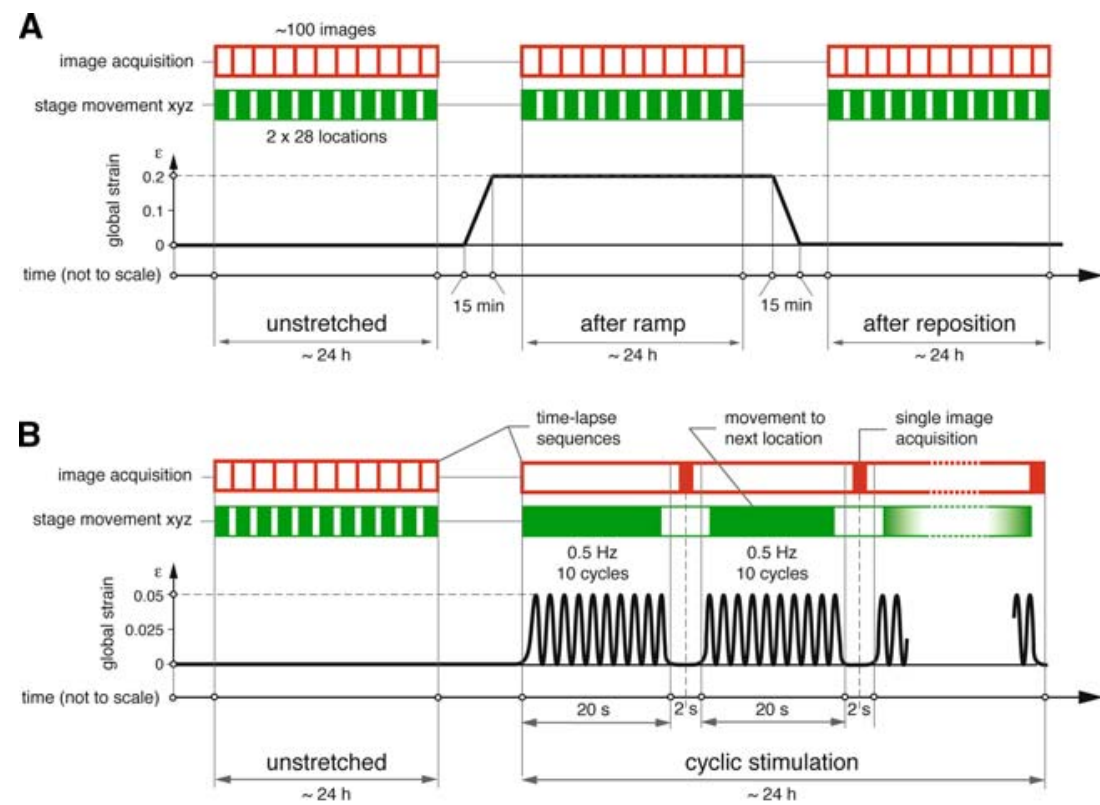

Fig. 1 Schematic diagram of the temporally coordinated mechanical stimulation (black), the stage movement (green), and the image acquisition (red). The global strain indicates the uniaxial elongation $\varepsilon=\Delta l / l_{0}$. A shows the ramp loading/unloading regime whereas in $\mathbf{B}$ the cyclic overloading regime is depicted (sinusoidal stimulation). Note nonlinearity of the time axis. The regime in $\mathbf{A}$ and $\mathbf{B}$ comprises three and

gelation and swelling. To enable the clamping of the very fragile cell-populated hydrogels to the carriages of the cell chamber, stainless steel meshes were cast into the hydrogel in a process similar to injection molding. Details on making the cell-seeded constructs can be found in the preceding companion paper (Ref to part I, Raeber et al. 2007a). The constructs were cultured under free-floating conditions for approximately $48 \mathrm{~h}$ before they were installed in the cell chamber for succeeding experiments.

\subsection{Digital time-lapse microscopy and analysis of single cell migration}

Cell migration of HFFs in PEG hydrogels was quantified from time-lapse sequences by a correlated random walk model as described in detail elsewhere (Raeber et al. 2005). Cells were classified as migrating if the persistence length of the migration path of the centroid was at least $2 \mu \mathrm{m}$. This criterion was selected based on the migration behavior of fibroblasts and based on the resolution and positioning precision of the measurement system. If too small a threshold is selected, then cell motion other than cell migration, e.g. cell jiggling with no long-term motion, or positioning imprecision can create the impression of migration when in fact there may be none. The length of cell tracks was between 15 and $20 \Delta t$ (time-lapse interval $\Delta t=15 \mathrm{~min}$ ). Total observation time was $20-25 \mathrm{~h}$. Cell speed and persistence time were $\log _{10}$ two time-lapse sequences, respectively. For one sequence $\sim 100$ images were taken with a time interval of $15 \mathrm{~min}$ from 28 locations in each of the two constructs. For simultaneous mechanical stimulation and timelapse imaging in regime $\mathbf{B}$, the stimulation unit was halted for $2 \mathrm{sec}$ every 10 cycles in the relaxed state and an image was acquired. During the subsequent stimulation cycle, the stage moved to the next location

transformed, resulting in close to normal distributed values and, therefore, allowing the application of parametric statistical tests. Equality of variances was proven. Significance was tested by pairwise Student's- $t$ tests using the Bonferroni correction to account for multiple comparisons. For statistical analysis of the nominal parameter migrating/nonmigrating, contingency analysis by multiple pairwise Pearson's Chisquare test was conducted ( $\alpha$-levels were again Bonferroni corrected).

Commonly, the mean-squared displacement $\left\langle d^{2}(t)\right\rangle$ is calculated from the projected (e.g. two-dimensional) displacement data of a single cell track and fitted to a correlated random walk model with a least squares regression algorithm (Parkhurst and Saltzman 1992). This procedure results in a scalar value for the persistence time, $P$, that describes the straightness of the migration path for that cell. In this work, migration parameters were determined in two orthogonal directions: along the $x$-axis in the principal direction of strain and along the $y$-axis perpendicular to it. By treating the displacement data in the $x$ - and the $y$-direction independently, one can acquire two persistence times, $P_{x}$ and $P_{y}$, along the $x$ - and the $y$-axis, respectively. These values are a measure for the uniformity of motion along these axes. To determine $P_{x}$ and $P_{y}$, the mean-squared displacements $\left\langle d_{x}^{2}(t)\right\rangle$ and $\left\langle d_{y}^{2}(t)\right\rangle$ along the two axes were computed. To that end, the coordinates of the position vector $r_{k}=$ $r\left(x_{k}, y_{k}\right)$ were used to calculate the displacement, $d$, for each 

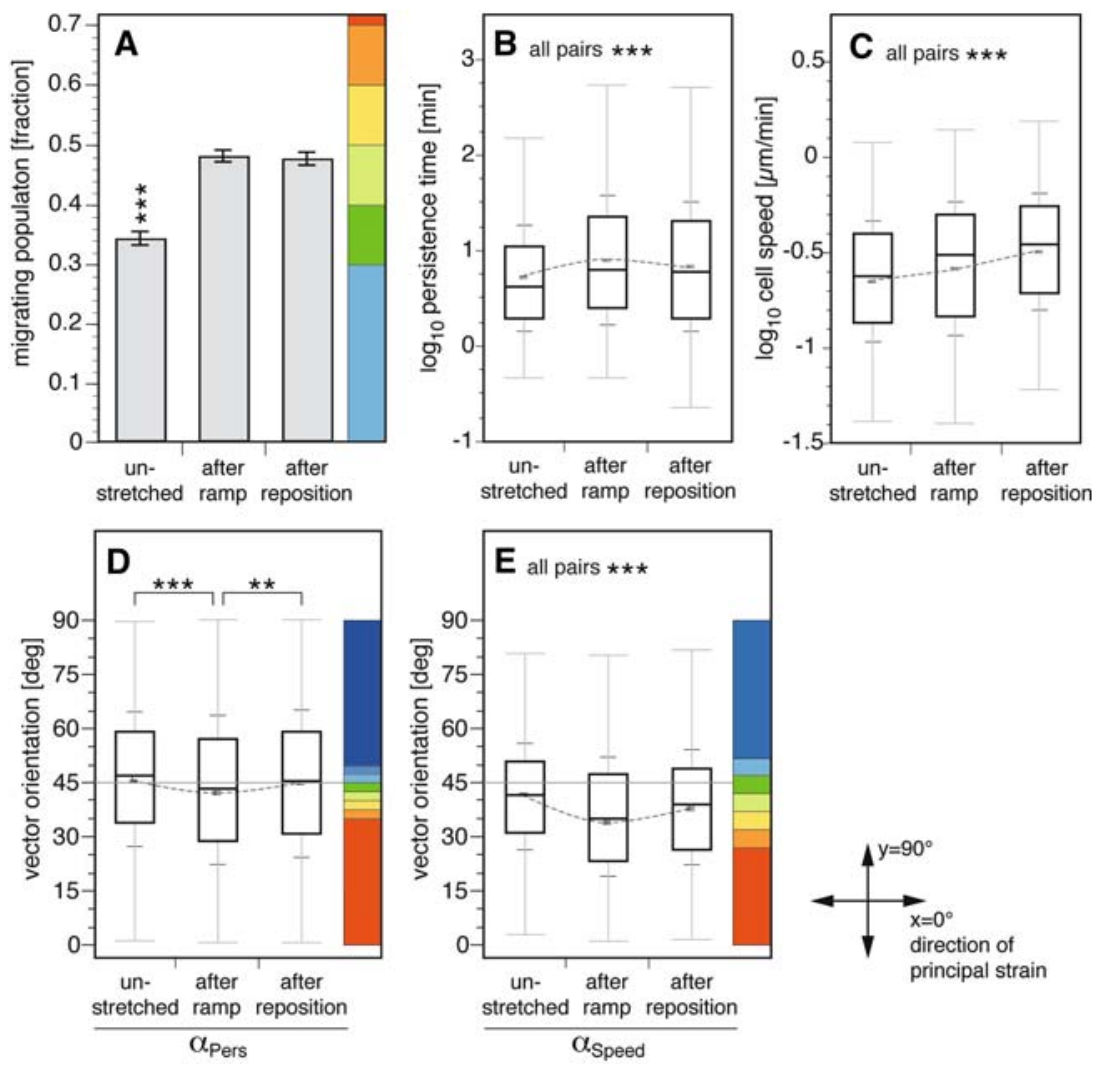

Fig. 2 Analysis of pooled migration parameters before (unstretched), while the sample was stretched by $20 \%$ (after ramp), and after restoration of the initial unstrained state (after reposition). The fraction of migrating cells (A) increased significantly while the construct was subjected to the exogenous strain and remained at the same level after strain release. Cells were classified as migrating if the persistence length of the migration path was at least $2 \mu \mathrm{m}$. Error bars in $\mathbf{A}$ represent estimates of the standard deviations for the group means assuming equal variances. Persistence time and speed ( $\mathbf{B}$ and $\mathbf{C}$ ) were $\log _{10}$ transformed to get close to normal distributed values. Strain induced a significant increase in the persistence time and speed of cellular migration. While the persistence time decreased to an intermediate level after strain release $(\mathbf{B})$, the cell speed further increased after reposition $(\mathbf{C})$. To determine a preferential direction of migration, vectorial migration data was analyzed along and

cell at each time point, $k$, over the time interval $t_{i} \equiv i \Delta t$ as follows:

$d_{x, i k}=x_{k+i}-x_{k}$,

$d_{y, i k}=y_{k+i}-y_{k}$.

The values $d_{x, i k}$ and $d_{y, i k}$ were considered as random variables with the expected squared values $\left\langle d_{x}^{2}(t)\right\rangle$ and $\left\langle d_{y}^{2}(t)\right\rangle$ given by

$\left\langle d_{x}^{2}(t)\right\rangle=2 \cdot S_{x}^{2} P_{x}\left[t-P_{x}\left(1-\mathrm{e}^{-t / P_{x}}\right)\right]$,

$\left\langle d_{y}^{2}(t)\right\rangle=2 \cdot S_{y}^{2} P_{y}\left[t-P_{y}\left(1-\mathrm{e}^{-t / P_{y}}\right)\right]$,

where $S_{x}$ and $S_{y}$ are the mean-squared cell speeds (see below). To obtain the measured mean-squared displacement for a perpendicular to the direction of principal strain ( $x$ and $y$, respectively). The analysis of the angle between the two vectors is shown in $\mathbf{D}$ and E. In strained constructs cells preferentially migrate in the direction of the principal strain. Upon strain release, this preference is only partially abrogated. The data in B-E is displayed as box-plots ranging from the 25 th to the 75th quantiles including the median and whiskers extending 1.5 times the interquartile range. The small vertical lines are centered on each group mean and give the standard error and the standard deviation of the group mean. Statistical analysis was done as described in Materials and Methods. ** $p<0.01$, *** $p<0.001$. In A, B, and $\mathbf{C}$ approximately 2,200 tracks were analyzed, in $\mathbf{D}$ and $\mathbf{E}$ data from 3,500 tracks is pooled. The color code on the right-hand side of $\mathbf{A}, \mathbf{D}$, and $\mathbf{E}$ corresponds to Figs. 3, 4, 5, respectively. The results are representative for four constructs of two independent experiments

given time interval $t_{\mathrm{i}}$, overlapping time intervals (Dickinson and Tranquillo 1993) were used to average all squared displacements $d_{x, i k}^{2}$ and $d_{y, i k}^{2}$ over the cell track of that interval size $t_{i} \equiv i \Delta t$ :

$$
\begin{aligned}
\bar{d}_{x, i}^{2} & =\frac{1}{n_{i}} \sum_{k=1}^{n_{i}} d_{x, i k}^{2}, \\
\bar{d}_{y, i}^{2} & =\frac{1}{n_{i}} \sum_{k=1}^{n_{i}} d_{y, i k}^{2}, \\
n_{i} & =N-i+1,
\end{aligned}
$$

where $N$ is the total number of time intervals comprising the cell track. The mean-squared cell speeds $S_{x}$ and $S_{y}$ were 


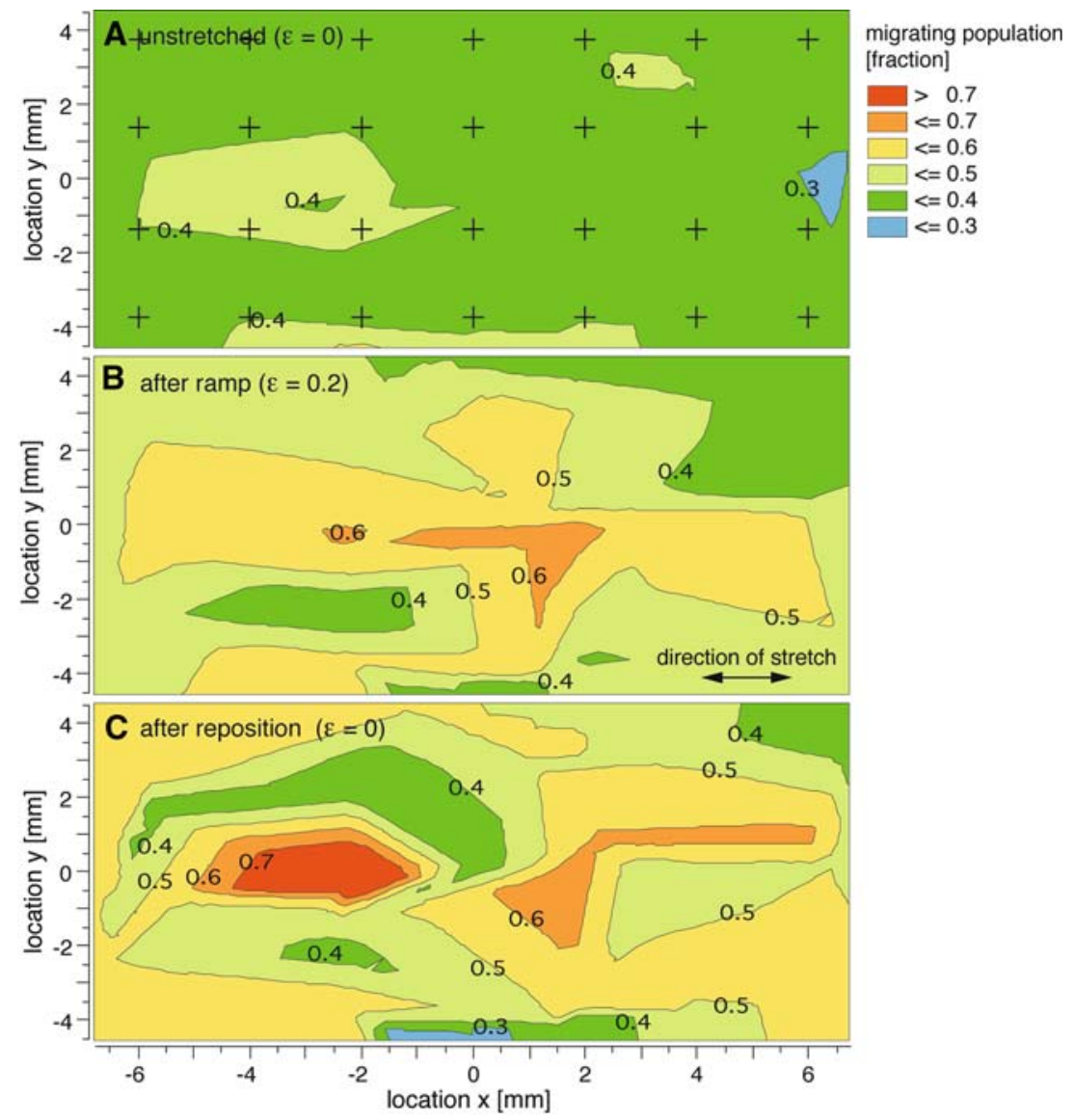

Fig. 3 Contour plots of the spatial distribution of the fraction of migrating cells for a representative construct before (A, unstretched), while the sample was stretched by $20 \%$ (B, after ramp), and after restoration of the initial unstrained state ( $\mathbf{C}$, after reposition). Cell migration data was assigned to the 28 image acquisition locations forming a grid as shown in $\mathbf{A}$. The track data was pooled for every location. The contour plots were made from a refined grid with interpolated data $(40 \times 25$ points). Areas between lines of equal values are color-coded. Cells were

calculated from the displacement data:

$S_{x}=\frac{\sqrt{\bar{d}_{x, 1}^{2}}}{\Delta t}$
$S_{y}=\frac{\sqrt{\bar{d}_{y, 1}^{2}}}{\Delta t}$

and than inserted into Eq. (2). This procedure was used to determine the orientation of persistence time, $\alpha_{\mathrm{Pers}}$ and the orientation of time, $\alpha_{\text {Speed }}$ for every cell track of a location within the construct:

$$
\begin{gathered}
\alpha_{\text {Pers }}=\arctan \left(\frac{P_{y}}{P_{x}}\right), \\
\alpha_{\text {Speed }}=\arctan \left(\frac{S_{y}}{S_{x}}\right) .
\end{gathered}
$$

classified as migrating if the persistence length of the migration path was at least $2 \mu \mathrm{m}$. In the unstretched construct, the fraction of migrating cells showed a very uniform distribution (A). The imposed strain increased the number of migrating cells markedly. Cells populating central areas were subjected to a more prominent augmentation in migration (B). After repositioning the overall number of migrating cells did not noticeably decrease but the distribution became less ordered. The results are representative for four constructs of two independent experiments

Since $S$ and $P$ are positive, $\alpha_{\text {Pers }}$ and $\alpha_{\text {Speed }}$ take values between $0^{\circ}$ and $90^{\circ}$. The $0^{\circ}$ orientation corresponds to the direction of principal strain (e.g. the direction of uniaxial stimulation). In an undisturbed (unbiased) setup, random motility leads to identical values of the persistence time along any direction and $\alpha_{\text {Pers }}=\alpha_{\text {Speed }}=45^{\circ}$. Conversely, in the case that cells migrate in the direction of the stimulation axis the angles $\alpha_{\text {Pers }}$ and $\alpha_{\text {Speed }}$ are smaller than $45^{\circ}$. The parameters were averaged for all tracks from the same acquisition location within the hydrogels. By assigning them to their acquisition coordinates, a scalar field was obtained. Comparing this scalar field with the data from the FE analysis, it was possible to correlate local migration behavior with locally estimated stresses and strains.

To introduce time dependence into the persistence time, $P$, and the cell speed, $S$, the calculation of the mean-squared 


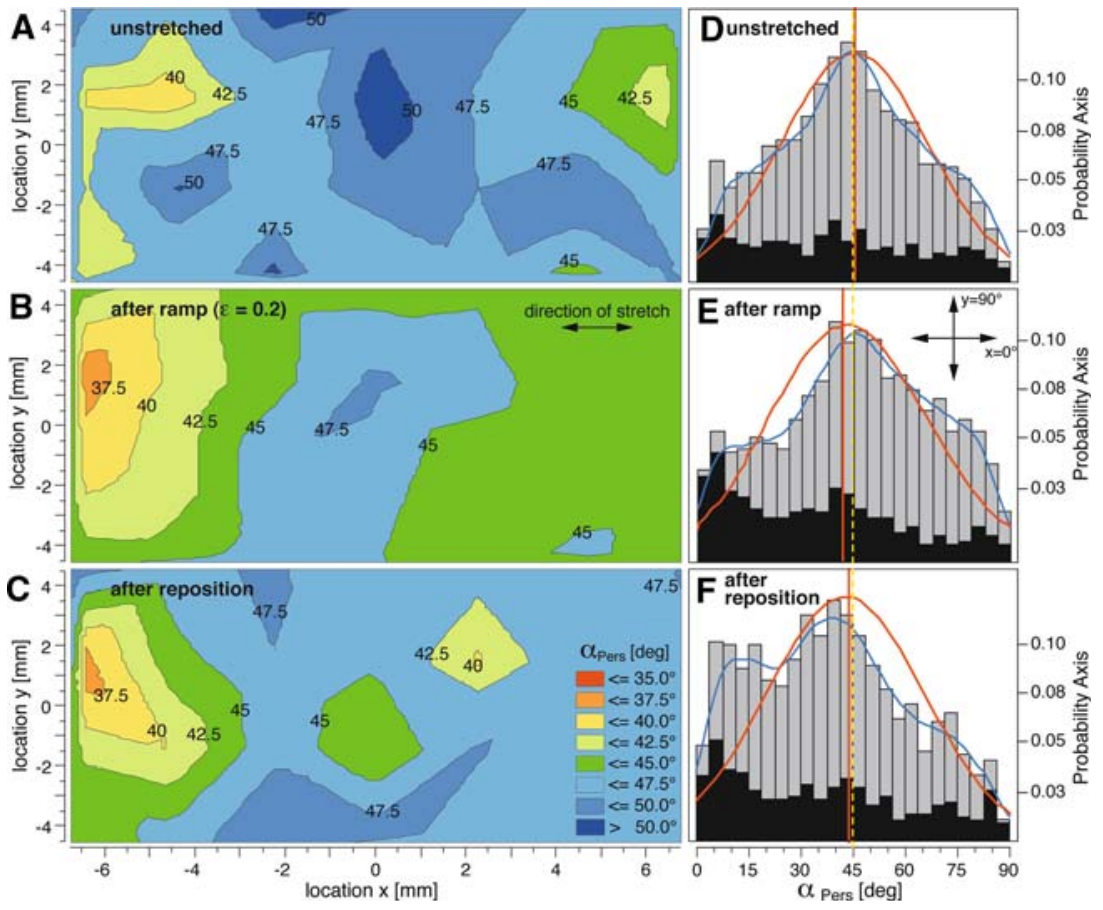

Fig. 4 (A-C) Contour plots of the spatial distribution of the persistence time orientation $\left(\alpha_{\text {Pers }}\right)$ for a representative construct before (A, unstretched), while the sample was stretched by $20 \%$ (B, after ramp), and after restoration of the initial unstrained state ( $\mathbf{C}$, after reposition). Cell migration data was assigned to the 28 image acquisition locations (see Fig. 3A). The track data was pooled for every location. The contour plots were made from a refined grid with interpolated data $(40 \times 25$ points). Areas between lines of equal values are color-coded. (D-F) Histograms showing the distribution of individual $\alpha_{\text {Pers }}$-values. Every track of the entire construct contributed with one value. The migrating population is shown in black. A normal distribution $(\mathrm{red})$ is fitted to the data, the density distribution is shown in blue, and a vertical red line indicates the mean. In the unstretched construct, the mean value for $\alpha_{\text {Pers }}$ was $45^{\circ}$

displacements, $\bar{d}_{x, i}^{2}$ and $\bar{d}_{y, i}^{2}$, was modified similar to a method described by (Shreiber et al. 2003).

\section{Results}

3.1 Static mechanical stimulation induces a sustained increase in migration in the direction of principal strain

The ability of the system to simultaneously quantify cell migration and mechanically stimulate cells embedded in a $3 \mathrm{D}$ construct was first tested with a prototypical static stimulation regime depicted in Fig. 1A. Strain induced an increase in the migratory activity of cells that was only partially abrogated after strain release (Fig. 2). While the constructs were strained, fibroblasts migrated preferentially in the direction of the principal strain, that is, along the $x$-axis. The persistence time was longer and the speed higher in that direction than perpendicular to it. Overall cell speed and persistence
(D) as anticipated for randomly migrating cells and the spatial distribution was uniform (A). $20 \%$ uniaxial strain (in the $x$-direction, $0^{\circ}$ ) induced a decrease of $\alpha_{\text {Pers }}(\mathbf{B})$ indicating that the persistence time and, therefore, the time during which the cells were migrating in the direction of principal strain was greater than perpendicular to it. The migrating population reacted to the strain with an increased frequency of small angles (see E). The associated contour plot shows that the alignment of the persistence time with the principal strain is strongest close to the left border (clamping region). After restoring the initial strain state (C and $\mathbf{F}$ ), the persistence time orientation was largely reestablished (see $\mathbf{F}$, mean $\alpha_{\text {Pers }}$ at $45^{\circ}$ ) however the spatial distribution remained disturbed (C)

time increased significantly compared to the unstrained state. After repositioning, the number of migrating cells did not diminish (Fig. 2A) but the cells migrated in a less ordered fashion. The imposed levels of strain were within the range of physiological strains for fibroblasts in a number of situations (Pedersen and Swartz 2005).

\subsection{Spatial and temporal resolution of migration parameters}

In a next step, the changes in migration parameters upon mechanical stimulation were spatially resolved (Figs. 3, 4, 5). The mechanical stimulus increased the number of migrating cells markedly (Fig. 3A). The distribution changed from very uniform to a pattern, indicating the center as the area subjected to the most pronounced increase in cell migration. Unloading did not decrease the overall percentage of migrating cells (Fig. 2A) but randomly redistributed the zones of increased migratory activity (Fig. 3C). The centers of migration activity seen in Fig. $3 \mathrm{~B}$ and $\mathrm{C}$ are several millimeters 


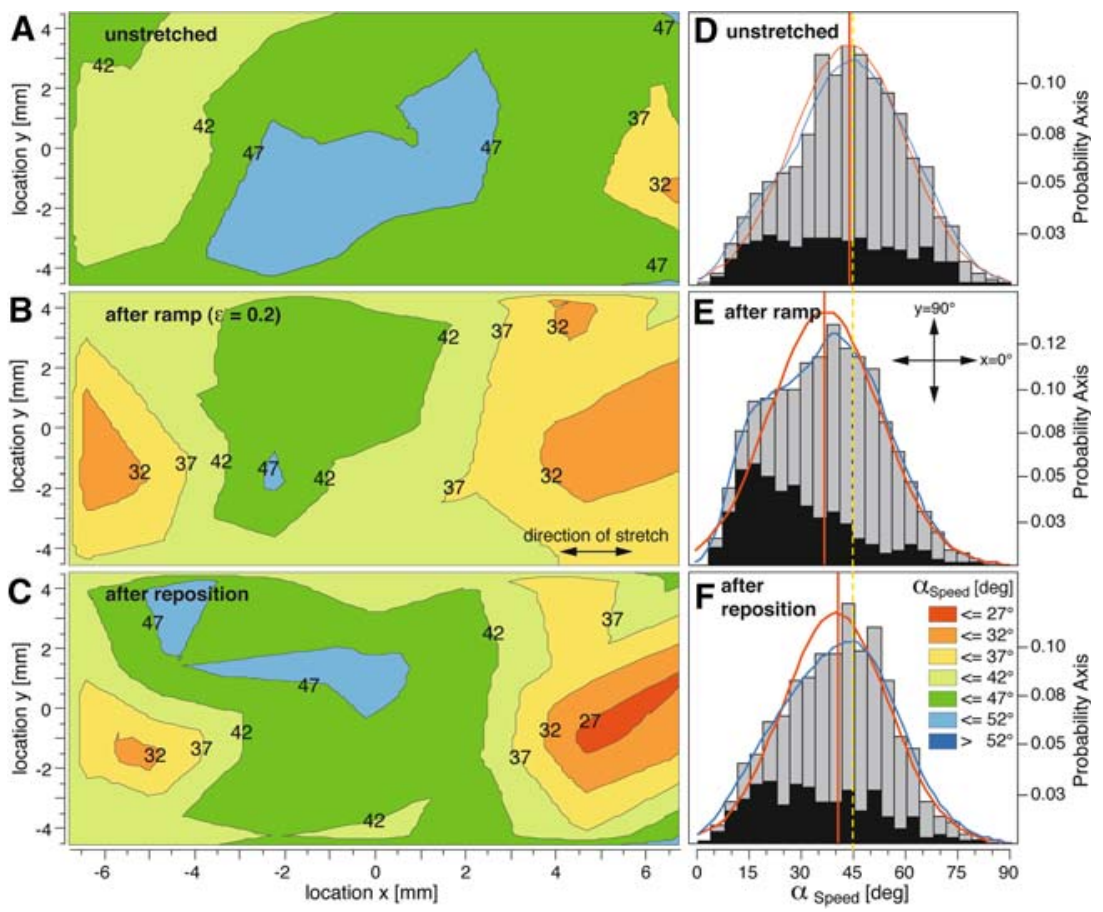

Fig. 5 (A-C) Contour plots of the spatial distribution of the cell speed orientation $\left(\alpha_{\text {Speed }}\right)$ for a representative construct before (A, unstretched), while the sample was stretched by $20 \%$ (B, after ramp), and after restoration of the initial unstrained state (C, after reposition). Cell migration data was assigned to the 28 image acquisition locations (see Fig. 3A). The track data was pooled for every location. The contour plots were made from a refined grid with interpolated data $(40 \times 25$ points). Areas between lines of equal values are color-coded. (D-F) Histograms showing the distribution of individual $\alpha_{\text {Speed }}$-values. Every track of the entire construct contributed with one value. The migrating population is shown in black. A normal distribution (red) is fitted to the data, the density distribution is shown in blue, and a vertical

apart and, therefore, not generated by the same subpopulation of stretch-activated cells that moved. Persistence time orientation and cell speed orientation of HFFs showed comparable changes in contour plots when the single loading/unloading regime was applied (Figs. 4, 5). Histograms of $\alpha_{\text {Pers }}$ and $\alpha_{\text {Speed }}$ illustrate an overall shift of the distribution to lower values upon mechanical stimulation. Unloading reestablishes the unstretched condition only partially. The black portion of the histograms correspond to the migrating subpopulation of HFFs and demonstrate that in particular these cells react strongly to a change in the mechanical environment (e.g. see Fig. 5E).

In general, a decrease in both angles ( $\alpha_{\text {Pers }}$ and $\left.\alpha_{\text {Speed }}\right)$ was observed, indicating faster and less tortuous migration along the principal strain axis than perpendicular to it. Interestingly, cells close to the clamping regions showed a stronger response than in the central region (e.g. see Fig. 5B). The contour plots had a certain similarity with the strain field that was determined by FE analysis in the preceding companion paper (Ref to part I, Raeber et al. 2007a). As mentioned red line indicates the mean. Similarly to the persistence time orientation, in the unstretched construct, the mean value for $\alpha_{\text {Speed }}$ was $45^{\circ}$ (D) and the spatial distribution was largely uniform (A). 20\% uniaxial strain (in the $x$-direction, $0^{\circ}$ ) decreased $\alpha_{\text {Speed }}(\mathbf{B})$, indicating that the cells were migrating preferentially in the direction of principal strain. The distribution of the $\alpha_{\text {Speed }}$-values of the migrating population was markedly shifted to smaller angles with a median of $\sim 15^{\circ}$ (black, $\mathbf{E}$ ). After repositioning, the central area of the construct recovered a random orientation of the speed vector $\left(\mathbf{C}, \alpha_{\text {Speed }}\right.$ at $\left.45^{\circ}\right)$, however, a preferential orientation of cell migration along the main axis of the construct remained in the clamping regions $\left(\mathbf{C}, \alpha_{\text {Speed }}<45^{\circ}\right)$

earlier, uniaxial stretch causes compression strains in the plane perpendicular to the principal strain axis. The FE model of the construct suggested that the compression strain in the $y$-axis was not maximal in the center of the construct but in a region close to the clamping region. Furthermore, the gradients of $\varepsilon_{x}$ and $\varepsilon_{z}$ were steepest in that region. These factors may have induced a more pronounced directional migration response close to the clamping region than the mechanical microenvironment in the center of the construct. So far, we did not investigate a spatially resolved net cell flux and can not give evidence whether cells moved away from or into certain areas of the strain field. However, the presented system would allow such quantitative experiments.

\subsection{Dynamic mechanical stimulation has less pronounced effects on fibroblast migration}

To further exploit the possibility of the UCSD system, a second prototypical stimulation regime was examined that comprised simultaneous cyclic stretching and image 

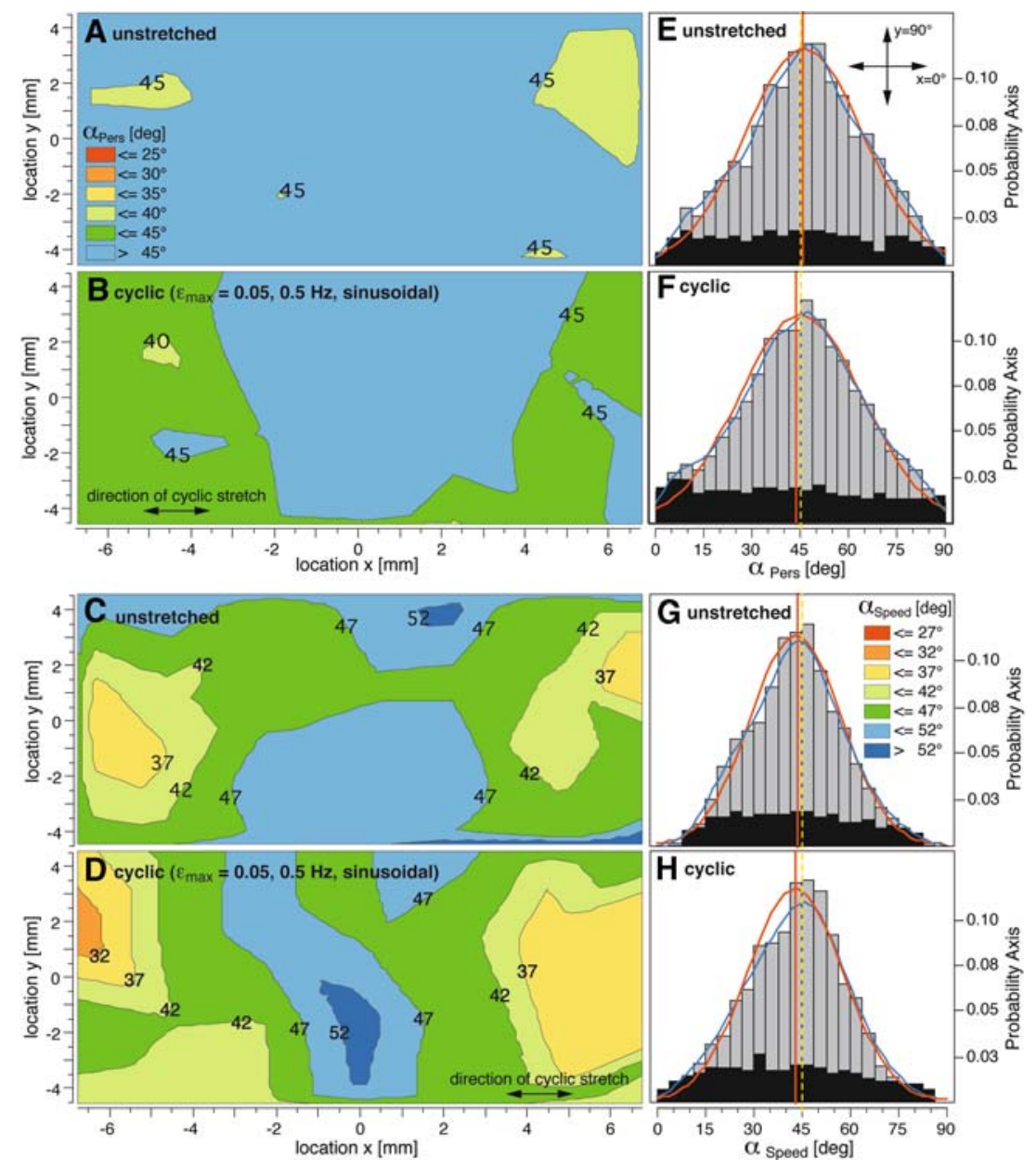

Fig. 6 Contour plots (A-D) and histograms $(\mathbf{E}-\mathbf{H})$ of the distribution of the persistence time orientation $\left(\mathbf{A}, \mathbf{B}, \mathbf{E}, \mathbf{F} ; \alpha_{\text {Pers }}\right)$ an the cell speed orientation $\left(\mathbf{C}, \mathbf{D}, \mathbf{G}, \mathbf{H} ; \alpha_{\text {Speed }}\right)$ for a representative construct before (unstretched) and during stimulation with $5 \%$ cyclic overload (cyclic). Cell migration data was assigned to the 28 image acquisition locations (see Fig. 3A). The track data was pooled for every location. The contour plots were made from a refined grid with interpolated data $(40 \times 25$ points $)$. Areas between lines of equal values are color-coded.

acquisition (see Fig. 1B). After acquiring a time-lapse sequence of the unstretched constructs, the samples were subjected to $5 \%$ cyclic overload with a frequency of $0.5 \mathrm{~Hz}$. Images from the different locations were acquired in 2-s windows every 10 cycles. Surprisingly, this regime did not alter the spatial distribution of $\alpha_{\text {Pers }}$ and $\alpha_{\text {Speed }}$ or the shape of the corresponding histograms as significantly as the static stimulation regime did (Fig. 6). However, over longer periods the fraction of migrating cells increased as well (Fig. 7). For the case discussed here, this may indicate that a sinusoidal 5\% cyclic stimulation had a more subtle effect than static $20 \%$ strain.

With the same data set, temporal resolution of migration was explored. Statistical migration parameters like the persistence time cannot be measured instantaneously (e.g. unlike
The migrating population in histograms is shown in black. A normal distribution $(r e d)$ is fitted to the data, the density distribution is shown in blue, and a vertical red line indicates the mean. The mean values for $\alpha_{\text {Pers }}$ and $\alpha_{\text {Speed }}$ were very close to $45^{\circ}$ (E and $\mathbf{G}$ ) and the spatial distribution was largely uniform (A and $\mathbf{C}$ ) in the unstretched construct, indicating random motility. The migratory response recorded during cyclic stimulation $(\mathbf{B}, \mathbf{F}, \mathbf{D}, \mathbf{H})$ showed the same tendency as after ramp loading (decrease of $\alpha_{\text {Pers }}$ and $\alpha_{\text {Speed }}$ ) but was less pronounced

the position) but may be determined for fixed interval periods. Figure 7 gives an example of the temporal evolution of the fraction of migrating cells. In static samples, the number of migrating cells remained constant during the observation period, whereas during cyclic stimulation a steady increase in migration was observed, which became statistically significant after $\sim 15 \mathrm{~h}$.

\section{Discussion}

Our finding that cells migrate preferentially in the direction of principal strain is supported by a number of other studies in a variety of model systems. Fibroblasts reoriented themselves in the direction of tensile stress when cuts rearranged the 

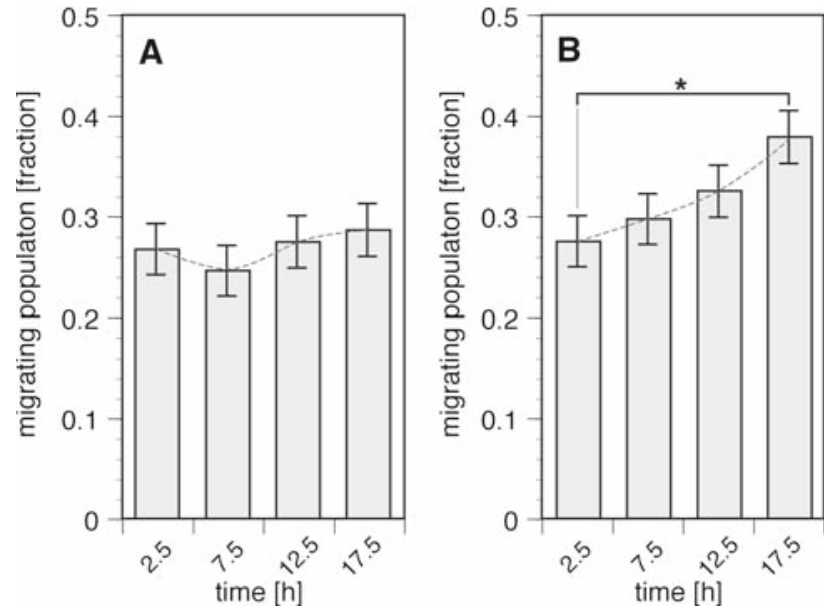

Fig. 7 Temporal evolution of the migrating HFF population in an unstimulated construct (A) and the same construct during stimulation with 5\% cyclic overload (B). The tracking length was restricted to 1520 time steps of $\Delta t=15 \mathrm{~min}$. The track start was binned into four groups of which the mid-values are indicated in the graphs. Approximately 800 tracks are included in each bin. Tracks were classified as migrating if the persistence length of the migration path was at least $2 \mu \mathrm{m}$. Error bars represent estimates of the standard deviations for the group means assuming equal variances. Cyclic stimulation increased the number of migrating cells. The trend became significant for longer observation times. $* p<0.05$

force balance in tethered collagen gels (Takakuda and Miyairi 1996). When the polyacrylamide substrate was locally pulled away from 3T3 cells, they formed or expanded lamellipodia in that direction and redirected their migration accordingly within an hour (Lo et al. 2000). Furthermore, the same cells migrated preferentially towards stiffer substrates. It was hypothesized that pulling away the membrane may be sensed by the cell equivalently to a stiffer substrate. Likewise, increased lamellipodia dynamics was observed at the corner of squared adhesive patches, where (due to the internal tension) local stress maxima may occur (Parker et al. 2002). Capillary sprouting was preferentially oriented in the direction of extension in a 3D collagen model (but not in fibrin) (Korff and Augustin 1999) and axonal growth was guided by externally applied mechanical tension (Bray 1984).

In this study, we have not examined the effects of stimuli other than an imposed strain, on cell migration. Elsewhere, we have reported the effects of potentially cell-secreted factors, such as cytokines, growth factors and MMPs on cell migration, which signals may relate to cell seeding density and cell-cell signaling (Raeber et al. 2007b).

\subsection{PEG hydrogels as model matrix in mechanobiology}

PEG hydrogels offer an interesting alternative to often used biopolymers as model matrices in mechanobiological investigations. Natural ECM-derived macromolecules that form a fibrillar meshworks like collagen or fibrin have mechanical properties with a considerable visco-elastic component that are responsible for transient relaxation phenomena (Knapp et al. 1997), which are difficult to control and may complicate the acquisition of long-term time-lapse sequences under static or dynamic mechanical stimulation. However, the almost ideal elastic behavior of PEG hydrogels enables re-detection of the exact same cells after many stimulation cycles and allows theoretical considerations based on linear elasticity theory. Furthermore, biopolymers allow only limited molecular modifications and possess a wealth of signaling molecules that are not controllable. An engineered synthetic matrix with only a few essential biological functionalities (e.g. adhesion ligands and protease substrates as used in this work), on the other hand, might reduce the complexity of the experimental model system considerably. The elastic modulus of PEG hydrogels can be adjusted from $G^{\prime}=10 \mathrm{~Pa}$ to $5 \mathrm{kPa}$ independently from adhesion ligand type or density (Lutolf and Hubbell 2003) and the degradability can be tailored to specific classes of enzymes (Raeber et al. 2005). These features of PEG hydrogels thus may enable one to target specific mechanobiological problems despite the simplicity of the matrix.

The type of PEG hydrogels used for these experiments restricts cell migration to MMP-dependent matrix remodeling (Lutolf et al. 2003b; Raeber et al. 2005). As a consequence, increased migration, as seen for mechanically stimulated constructs, possibly involves the upregulation of MMPs and/or their more efficient use in the pericellular environment. In fact, this view is supported by results from other studies: it has been shown that cyclic loading of fibroblasts in collagen increased their MMP-2 and MMP-9 synthesis and partially the activation of these enzymes (Prajapati et al. 2000a,b). MMP-2 plays an important role in pericellular proteolysis in that it can associate with integrins that bind to the ligands presented by the synthetic matrix (MMP-2 associates with $\alpha_{\mathrm{v}} \beta_{3}$ without hindering the binding of RGD; Brooks et al. 1996). Therefore, our results may suggest that fibroblasts are capable of adjusting their pericellular proteolytic machinery in response to the surrounding strain field.

In conclusion, our experiments show that high reposition accuracy of the stimulation unit, precise motion of the microscopy stage, favorable mechanical properties of the 3D cell carrier (e.g. linear elasticity, negligible creep, stable mesh-hydrogel bonding), high level of motion and vision integration, and state-of-the-art image analysis of time-lapse sequences permitted the spatial and temporal quantification of single cell migration under static and during dynamic mechanical stimulation. The understanding of how the dynamic mechanical extracellular environment and cell migration are linked provides potentially important insights into the control of soft tissue architecture during morphogenesis and regeneration. 


\section{References}

Balaban NQ, Schwarz US, Riveline D, Goichberg P, Tzur G, Sabanay I, Mahalu D, Safran S, Bershadsky A, Addadi L, Geiger B (2001) Force and focal adhesion assembly: a close relationship studied using elastic micropatterned substrates. Nat Cell Biol 3:466-472

Bray D (1984) Axonal growth in response to experimentally applied mechanical tension. Dev Biol 102:379-389

Brooks PC, Stromblad S, Sanders LC, von Schalscha TL, Aimes RT, Stetler-Stevenson WG, Quigley JP, Cheresh DA (1996) Localization of matrix metalloproteinase MMP-2 to the surface of invasive cells by interaction with integrin alpha $\mathrm{v}$ beta 3 . Cell 85:683-693

Chen CS, Mrksich M, Huang S, Whitesides GM, Ingber DE (1997) Geometric control of cell life and death. Science 276:1425-1428

Choquet D, Felsenfeld DP, Sheetz MP (1997) Extracellular matrix rigidity causes strengthening of integrin-cytoskeleton linkages. Cell 88:39-48

Cukierman E, Pankov R, Stevens DR, Yamada KM (2001) Taking cellmatrix adhesions to the third dimension. Science 294:1708-1712

Cukierman E, Pankov R, Yamada KM (2002) Cell interactions with three-dimensional matrices. Curr Opin Cell Biol 14:633-639

Dickinson RB, Tranquillo RT (1993) Optimal estimation of cell movement indices from the statistical analysis of cell tracking data. AIChE J 39:1995-2010

Duncan R, Turner C (1995) Mechanotransduction and the functional response of bone to mechanical strain. Calcif Tissue Int 57:344 358

Engler AJ, Sen S, Sweeney HL, Discher DE (2006) Matrix elasticity directs stem cell lineage specification. Cell 126:677-698

Geiger B, Bershadsky A (2001) Assembly and mechanosensory function of focal contacts. Curr Opin Cell Biol 13:584-592

Goldmann WH (2002) Mechanical aspects of cell shape regulation and signaling. Cell Biol Int 26:313-317

Henderson JH, Carter DR (2002) Mechanical induction in limb morphogenesis: the role of growth-generated strains and pressures. Bone 31:645-653

Hern DL, Hubbell JA (1998) Incorporation of adhesion peptides into nonadhesive hydrogels useful for tissue resurfacing. J Biomed Mater Res 39:266-276

Ingber DE (1997) Tensegrity: the architectural basis of cellular mechanotransduction. Annu Rev Physiol 59:575-599

Ingber DE (2002) Mechanical signaling and the cellular response to extracellular matrix in angiogenesis and cardiovascular physiology. Circ Res 91:877-887

Katsumi A, Orr AW, Tzima E, Schwartz MA (2004) Integrins in mechanotransduction. J Biol Chem 279:12001-12004

Katz BZ, Zamir E, Bershadsky A, Kam Z, Yamada KM, Geiger B (2000) Physical state of the extracellular matrix regulates the structure and molecular composition of cell-matrix adhesions. Mol Biol Cell 11:1047-1060

Knapp DM, Barocas VH, Moon AG, Yoo K, Petzold LR, Tranquillo RT (1997) Rheology of reconstituted type I collagen gel in confined compression. J Rheol 41:971-993

Koo LY, Irvine DJ, Mayes AM, Lauffenburger DA, Griffith LG (2002) Co-regulation of cell adhesion by nanoscale RGD organization and mechanical stimulus. J Cell Sci 115:1423-1433

Korff T, Augustin HG (1999) Tensional forces in fibrillar extracellular matrices control directional capillary sprouting. J Cell Sci 112:3249-3258

Li C, Wernig F, Leitges M, Hu Y, Xu Q (2003) Mechanical stress-activated PKCdelta regulates smooth muscle cell migration. FASEB J 17:2106-2108
Lo CM, Wang HB, Dembo M, Wang YL (2000) Cell movement is guided by the rigidity of the substrate. Biophys J 79:144-152

Lutolf MP, Hubbell JA (2003) Synthesis and physicochemical characterization of end-linked poly(ethylene glycol)-co-peptide hydrogels formed by Michael-type addition. Biomacromolecules 4:713-722

Lutolf MP, Hubbell JA (2005) Synthetic biomaterials as instructive extracellular microenvironments for morphogenesis in tissue engineering. Nat Biotechnol 23:47-55

Lutolf MP, Lauer-Fields JL, Schmoekel HG, Metters AT, Weber FE, Fields GB, Hubbell JA (2003a) Synthetic matrix metalloproteinase-sensitive hydrogels for the conduction of tissue regeneration: engineering cell-invasion characteristics. Proc Natl Acad Sci USA 100:5413-5418

Lutolf MP, Raeber GP, Zisch AH, Tirelli N, Hubbell JA (2003b) Cellresponsive synthetic hydrogels. Adv Mater 15:888-892

Parker KK, Brock AL, Brangwynne C, Mannix RJ, Wang N, Ostuni E, Geisse NA, Adams JC, Whitesides GM, Ingber DE (2002) Directional control of lamellipodia extension by constraining cell shape and orienting cell tractional forces. FASEB J 16:1195-1204

Parkhurst MR, Saltzman WM (1992) Quantification of human neutrophil motility in three-dimensional collagen gels. Effect of collagen concentration. Biophys J 61:306-315

Pedersen JA, Swartz MA (2005) Mechanobiology in the third dimension. Ann. Biomed. Eng. 33:1469-1490

Pierschbacher MD, Ruoslahti E (1984) Cell attachment activity of fibronectin can be duplicated by small synthetic fragments of the molecule. Nature 309:30-33

Prajapati RT, Chavally-Mis B, Herbage D, Eastwood M, Brown RA (2000a) Mechanical loading regulates protease production by fibroblasts in three-dimensional collagen substrates. Wound Repair Regen 8:226-237

Prajapati RT, Eastwood M, Brown RA (2000b) Duration and orientation of mechanical loads determine fibroblast cyto-mechanical activation: monitored by protease release. Wound Repair Regen 8:238-246

Raeber GP, Mayer J, Hubbell JA (2007a) Part I: A novel in vitro system for simultaneous stimulation and time-lapse microscopy in 3D. Biomech. Model Mechanobiol (in press)

Raeber GP, Lutolf MP, Hubbell JA (2007b) Mechanisms of 3D migration and matrix remodeling of fibroblasts within artificial ECMs. Acta Biomater (in press)

Raeber GP, Lutolf MP, Hubbell JA (2005) Molecularly engineered PEG hydrogels: a novel model system for proteolytically-mediated cell migration. Biophys J 89:1374-1388

Riveline D, Zamir E, Balaban NQ, Schwarz US, Ishizaki T, Narumiya S, Kam Z, Geiger B, Bershadsky AD (2001) Focal contacts as mechanosensors: externally applied local mechanical force induces growth of focal contacts by an mDial-dependent and ROCKindependent mechanism. J Cell Biol 153:1175-1186

Sachs F, Morris CE (1998) Mechanosensitive ion channels in nonspecialized cells. Rev Physiol Biochem Pharmacol 132:1-77

Shreiber DI, Barocas VH, Tranquillo RT (2003) Temporal variations in cell migration and traction during fibroblast-mediated gel compaction. Biophys J 84:4102-4114

Shyy JY, Chien S (1997) Role of integrins in cellular responses to mechanical stress and adhesion. Curr Opin Cell Biol 9:707-713

Takakuda K, Miyairi H (1996) Tensile behaviour of fibroblasts cultured in collagen gel. Biomaterials 17:1393-1397

Tomasek JJ, Gabbiani G, Hinz B, Chaponnier C, Brown RA (2002) Myofibroblasts and mechano-regulation of connective tissue remodelling. Nat Rev Mol Cell Biol 3:349-363

Van Essen DC (1997) A tension-based theory of morphogenesis and compact wiring in the central nervous system. Nature 385:313318 
von Offenberg Sweeney N, Cummins PM, Birney YA, Redmond EM, Cahill PA (2004) Cyclic strain-induced endothelial MMP-2: role in vascular smooth muscle cell migration. Biochem Biophys Res Commun 320:325-333
Wang N, Butler JP, Ingber DE (1993) Mechanotransduction across the cell surface and through the cytoskeleton. Science 260:1124-1127 Williams B (1998) Mechanical influences on vascular smooth muscle cell function. J Hypertens 16:1921-1929 